\title{
DIFUSIÓN DE LA INVESTIGACIÓN CIENTÍFICA DE TURISMO EN CHILE Y ARGENTINA
}

\author{
Patricia Picazo Peral \\ Universidad de La Palmas Gran Canaria \\ Instituto de Turismo y Desarrollo Económico Sostenible. \\ España \\ patriciatides@gmail.com \\ Sergio Moreno Gil \\ Universidad de La Palmas Gran Canaria \\ Instituto de Turismo y Desarrollo Económico Sostenible. \\ España \\ smoreno@dede.ulpgc.es
}

\section{RESUMEN}

Teniendo en cuenta la relevancia del sector turístico en Argentina y Chile y las posibilidades de expansión y desarrollo que presentan los destinos, es notoria la necesidad de analizar la investigación turística que los investigadores e instituciones académicas realizan en el ámbito turístico. El presente artículo analiza la contribución realizada por aquellos investigadores afiliados a instituciones argentinas y chilenas en revistas académicas especializadas en turismo, tanto de ámbito nacional, como internacional. El análisis permite identificar aquellas instituciones y autores que han realizado un mayor esfuerzo de investigación y difusión en turismo. El artículo presenta no sólo un ranking de instituciones y autores, sino que también analiza el ámbito geográfico de la aplicación de las investigaciones, el número de coautores medio por trabajo, las citas de los trabajos, las revistas o publicaciones específicas en las que publican más activamente sus trabajos y la evolución temporal del resultado obtenido en la investigación turística. Finalmente, se establecen una serie de conclusiones en relación a la situación actual de la investigación en turismo en Argentina y Chile.

Palabras Claves: investigación, turismo, Chile, Argentina, universidad, ranking, revistas, publicación 


\title{
DISSEMINATION OF THE SCIENTIFIC RESEARCH OF TOURISM IN CHILE AND ARGENTINA
}

\author{
Patricia Picazo Peral \\ Universidad de La Palmas Gran Canaria \\ Instituto de Turismo y Desarrollo Económico Sostenible. \\ España \\ patriciatides@gmail.com \\ Sergio Moreno Gil \\ Universidad de La Palmas Gran Canaria \\ Instituto de Turismo y Desarrollo Económico Sostenible. \\ España \\ smoreno@dede.ulpgc.es
}

\begin{abstract}
Taking into account the relevance of the tourism sector in Argentina and Chile, the possibilities of expansion and development of its destinations, For this reason it is necessary to analyze the tourism research that the researchers and academic institutions in the field of tourism. This article analyzes the contribution made by those researchers affiliated with Argentine and Chilean institutions in academic journals in tourism, at both the national and international levels. The analysis allows us to identify those institutions and authors that have made a greater effort of research and dissemination in tourism. The paper not only present a ranking of institutions and authors, but also analyzes the geographic scope of the application of the research, the average number of coauthors by research, citation from your work, specific journals or publications you publish more work actively and temporal evolution of the results obtained in tourism research. Finally, laying down a series of conclusions in relation to the current state of research in tourism in Argentina and Chile.
\end{abstract}

Key words: research, tourism, Chile, Argentina, university, ranking, journals and publication 


\section{INTRODUCCIÓN}

Argentina y Chile son dos destinos turísticos de gran relevancia internacional con un protagonismo destacado en Sudamérica. En Argentina el desarrollo turístico juega un papel fundamental, siendo concebido como una política de Estado. Así lo demuestran las cifras record de 5,6 Millones de turistas internacionales que recibió el país en el año 2011, generando un ingreso de 5,3 millones de dólares según la Organización Mundial del Turismo (2012). A su vez, la actividad turística en Chile ha experimentado un gran aumento durante los últimos años, llegando recibir en el año 2011 a 3 millones de turistas extranjeros (OMT, 2012), lo que supone un aumento del 11\% respecto al año anterior. Pese a los buenos resultados y las posibilidades de desarrollo que ofrece el sector, las investigaciones realizadas en regiones con diferentes contextos culturales, como es el caso de Latinoamérica, y en especial la difusión de las mismas han sido prácticamente inexistentes (Svensson, Svaeri y Einarsen, 2009). En particular, la investigación turística en Chile sigue sin consolidarse, existiendo a su vez una clara carencia de información con respecto a la investigación turística Argentina (Castelluci, 2001).

Actualmente, existe un claro consenso en la comunidad académica en torno a la importancia de la investigación turística (Law y Chon, 2007; Sheldon, 1991) ya que permite divulgar y ampliar conocimientos sobre un sector que necesita adaptarse y anticiparse a los constantes cambios del entorno. La tendencia actual de expansión y globalización de la investigación hace que éste sea el periodo idóneo para la realización de evaluaciones en torno a las publicaciones académicas (Severt, Tesone, Bottorff y Carpenter, 2009), las cuales representan el principal canal de comunicación de los investigadores (Xiao y Smith, 2006). En este sentido, los autores que consiguen publicar sus investigaciones en revistas científicas juegan un papel fundamental como principales actores en la transmisión de conocimientos turísticos (Frechtling, 2004).

Como subrayan Pechlaner et al. (2004) y Law, Ye, Chen y Leung (2009), las publicaciones académicas son vitales para transmitir los hallazgos académicos que formarán los pilares de conocimientos de la disciplina. La divulgación de las investigaciones es esencial ya que ejerce una influencia directa en el desarrollo personal y trayectoria profesional de los investigadores (Bowen, 2005). En particular, los autores e instituciones académicas hacen uso de los factores de impacto de las publicaciones y del número de citaciones realizadas del artículo como herramienta indispensable en la medición de la productividad de su personal (Jamal et al., 2008; Rivera y Upchurch, 2008), siendo la cantidad y calidad de las investigaciones publicadas determinantes para el reconocimiento internacional de las universidades y de sus programas educativos (Bowen, 2005; Jogaratman, Chon, McCleary, Mena y Yoo, 2005; Zhoe y Ritchie, 2005; Park et al., 2011).

Este análisis permite identificar varios aspectos clave para comprender la situación actual global de la investigación turística en Argentina y Chile y su evolución en los últimos seis años (2006-2011): (1) identificar las instituciones y autores más activos y 
destacados, (2) identificar el destino o zona de aplicación de las investigaciones realizadas, (3) identificar las revistas o publicaciones en las que los investigadores argentinos y chilenos difunden actualmente su trabajo, y (4) analizar el número de autores medio por trabajo publicado y los trabajos más citados. Esta información es de gran utilidad para comprender de forma objetiva la situación actual de la investigación en estos países, y extraer conclusiones al respecto. Por este motivo, se analiza de forma independiente la contribución realizada en revistas internacionales de Iberoamérica, y las contribuciones en revistas internacionales de habla inglesa, junto con un agregado final.

El presente trabajo es de utilidad para los diferentes colectivos. Así, ofrece a los autores un marco de referencia para la mejor publicación y difusión de sus investigaciones sobre turismo; a los alumnos de doctorado una valoración de las instituciones referentes en investigación, y al sector turístico y la sociedad en su conjunto una valoración de la aportación actual y potencial de las diferentes instituciones.

A continuación, se realiza una revisión de la literatura en torno al análisis y evaluación de la productividad científica de los académicos, seguidamente se plantea la metodología, incluyendo una descripción detallada de la muestra seleccionada y de los procedimientos seguidos en la recolección de datos. Posteriormente se presentan un conjunto de tablas y rankings que exponen los resultados obtenidos en el periodo analizado, finalizando con las conclusiones del estudio, sus implicaciones y futuras líneas de investigación.

\section{Estado del Arte}

En la última década, se han llevado a cabo un gran número de investigaciones centradas en la evaluación de las publicaciones realizadas en revistas científicas del sector turístico. Los aspectos objeto de estudio son variados y ofrecen información relevante acerca de la productividad y eficiencia de los autores, universidades y países. Sheldon (1991) fue la pionera en evaluar la productividad de los autores y, en su estudio analizó las tendencias de la investigación turística y clasificó a los académicos según su productividad y área geográfica. En esta línea, un gran número de autores (e.g., Mathieu y McConomy, 2003; Jogaratman et al., 2005) han seguido su estela. Así, Severt et al. (2009) desarrollaron un análisis longitudinal donde analizaron las instituciones y áreas geográficas líderes en la investigación turística. Este estudio destaca que sólo el 1.10\% de las aportaciones analizadas fueron realizadas por autores Latinoamericanos, siendo la revista “The International Journal of Contemporary Hospitality Management” la más popular entre estos autores ya que publicó el $66.7 \%$ de sus investigaciones, seguida de "Annals of Tourism Research", "Journal of Travel Research", "Journal of Hospitality and Leisure Marketing” y "The Journal of Travel and Tourism Marketing”. Park et al. (2011) por su parte, examinaron las contribuciones individuales y colectivas realizadas por autores internacionales entre los años 2000 y 2009 en las seis publicaciones de "Hospitality" y turismo con mayor reconocimiento a nivel mundial, y entre los 100 países 
que han contribuido más activamente al desarrollo de conocimiento de estas áreas, entre los países iberoamericanos sólo se encuentra España en el quinto puesto, Portugal en el vigesimoquinto y Brasil en el trigésimo.

Los análisis descriptivos sobre la productividad de las instituciones y de los líderes académicos motivaron la investigación acerca de otros tópicos relacionados como el liderazgo académico en turismo (Bowen, 2005; Zhao y Ritchie, 2007; Ladkin y Weber, 2008; y Law, Leung, Buhalis, 2010), los factores que influyen en la producción académica (Chung y Petrick, 2011; y Lee y Law, 2011), la evaluación del impacto de la investigación turística (Law et al., 2009), y en algunos casos cuestionado la validez de la metodología utilizada en la medición de la productividad (Page, 2005; Ryan, 2005; Jamal et al., 2008; y Hall, 2011).

\section{METODOLOGÍA}

El proceso de selección de la muestra de revistas que formarán parte de la investigación es un tema controvertido (Albacete Sáez y Fuentes Fuentes, 2010), el cual requiere una especial atención (Park et al., 2011). Hasta la fecha no disponemos de métodos infalibles o de estadísticas oficiales que midan la relevancia de las revistas del sector y faciliten su selección (McKercher, Law, Lam, 2006; Zhao y Ritchie, 2007), aunque existen diferentes procedimientos que permiten obtener una muestra representativa que cumpla con los objetivos planteados por el estudio. Entre ellos se encuentran la revisión de la literatura, las consultas a profesionales del sector, el análisis de los índices de impacto de cada publicación o el volumen de citas recibidas.

A la hora de realizar la selección final de publicaciones se tuvo en consideración las investigaciones más relevantes en el área, las aportaciones realizadas por profesionales del sector, el periodo de análisis disponible y nuestras reflexiones personales. Aunque la elección de las publicaciones es en cierto grado subjetiva, tres factores justifican nuestra selección (Zhao y Ritchie, 2007): Por un lado todas las revistas son reconocidas internacionalmente, segundo, la muestra ha sido seleccionada de tal modo que permitiese observar la gran variedad de materias que engloba la industria turística y tercero todas las revistas llevan como mínimo seis años realizando publicaciones periódicas. Todo ello dio lugar al análisis de 31 Revistas que se muestran en la tabla 1 (15 Internacionales de habla inglesa, 6 españolas, 7 brasileñas, 1 chilena, 1 argentina y 1 mexicana) identificándose un total de 2020 artículos realizados por instituciones iberoamericanas en estas revistas. Se estableció un periodo de investigación de 6 años, comenzando en 2006 y finalizando en 2011, donde todos los artículos de cada uno de los volúmenes y ediciones fueron revisados. Este marco temporal ofrece la representatividad y actualidad necesaria para el cumplimiento de los objetivos planteados. También se decidió incorporar con carácter informativo revistas que no cumpliesen estrictamente este requisito, ya que otorgaban riqueza y valor añadido al estudio. 


\section{Tabla 1. Muestra de revistas analizadas.}

\section{Internacionales de habla inglesa}

Annals of TourismResearch

ATR

Cornell Hospitality Quarterly

CHQ

International Journal of Contemporary Hospitality Management

IJCHM

International Journal of Hospitality Management

IJHM

International Journal of Tourism Research

IJTR

Journal of Hospitality \& Tourism Research

JHTR

Journal of Hospitality Marketing \& Management *

JHMM

Journal of Sustainable Tourism

JST

Journal of Travel \& Tourism Marketing *

JTTM

Journal of Travel Research

JTR

Journal of Vacation Marketing

JVM

Tourism Analysis

TA

Tourism Economics *

TE

Tourism Geographies

TG

Tourism Management

TM

\section{Españolas}

Análisis turístico

ANÁL. TUR.

Cuadernos de Turismo *

CUAD. TUR.

Estudios Turísticos

ESTUD. TUR.

Papers de Turisme

PAPERS

Pasos *

PASOS

RESTMA

RESTMA

\section{Brasileñas}

Caderno Virtual de Turismo *

CVT

Revista Brasileira de Pesquisa em Turismo*

RBTUR

Revista de Cultura e Turismo

CULTUR

Revista Observatório de Inovação do Turismo *

OIT

Revista Turismo emAnálise *

TUR. ANÁL.

Turismo - Visão e Ação *

VISAO E AÇAO

Turismo e Sociedade *

TUR. SOC. 


\section{Otras Latinoamericanas}

El Periplo Sustentable *

PSUS

Estudios y Perspectivas en Turismo *

ESTUD. PERSP.

TUR.

Gestión Turística *

GESTUR

* Revistas con producción de autores afiliados a instituciones argentinas y chilenas entre 2006-2011.

Una vez escogida la muestra, siguiendo la segmentación realizada por Mathieu y McConomy (2003), se seleccionaron aquellos artículos que hubieran sido publicados por al menos un investigador perteneciente en el momento de la publicación a instituciones académicas argentinas y chilenas, excluyendo de la muestra a aquellos autores que siendo argentinos o chilenos se encuentren afiliados a universidades extranjeras no incluidas en nuestra selección.

Con el objetivo de cumplir los objetivos marcados y de obtener datos homogéneos, fueron considerados únicamente los artículos y las notas de investigación, dejando al margen las reseñas de libros, las editoriales, artículos de discusión, opiniones y ensayos, introducciones y publicaciones similares. Este estudio utilizará una técnica de conteo basada en la información pública disponible sobre los artículos publicados en 31 revistas de "hospitality" y turismo. La aplicación del método del conteo de las publicaciones, ofrece como afirman Law et al. (2010) un incentivo para los académicos e instituciones, además de permitir calcular la productividad desde un punto de vista objetivo.

En cuanto a la afiliación académica de los autores, uno de los objetivos de nuestro estudio, se seleccionó aquella universidad o centro de investigación donde el autor desarrollase su actividad profesional en el momento de la publicación. Por regla general las revistas internacionales muestran de forma precisa la universidad de pertenencia de los autores, pero en el caso de otras publicaciones con diferente estructura editorial se hace referencia a la extensa biografía del investigador, en este caso se escogió la universidad donde realizaba actividades docentes y en su defecto aquella institución académica donde realizase sus estudios.

La recolección de los datos se realizó en los meses de Agosto y Diciembre de 2011, accediendo a las revistas seleccionadas desde su página Web o a través de recursos electrónicos como SCOPUS, EBSCO, Elsevier y Dialnet. Para cada uno de los artículos se seleccionaron como variables de estudio el título de la revista, el título del artículo, el nombre del autor, la afiliación del autor, país, año de publicación, volumen y palabras clave. 
A la hora de identificar y evaluar la calidad de los artículos publicados surge la dificultad de encontrar un método único que permita su medición de manera objetiva (Law et al., 2010, Lee y Law, 2011). Recientemente se ha incrementado la importancia y popularidad del análisis de citas como uno de los mejores indicadores de la difusión y utilización del conocimiento (Xiao y Smith, 2008; Law et al., 2009). Este método, que parte de la premisa de que los autores sólo citarán aquellos artículos que consideren realmente importantes para el desarrollo de su investigación (Pechlaner et al., 2004; Severt et al., 2009), permitirá contabilizar el número de veces en que un artículo ha sido citado por otra publicación, dotando de información objetiva sobre su calidad e influencia (Mathieu y McConomy, 2003).

La base de datos bibliográfica SCOPUS ha sido tradicionalmente utilizada como uno de los principales recursos en el análisis del impacto de las investigaciones turísticas (Xiao y Smith, 2008) debido a la amplia cobertura ofrecida sobre revistas científicas del sector (Hall, 2011), sin embargo su importancia ha disminuido en los últimos años debido al desarrollado e implantación de otras herramientas similares como Google Scholar, que permite realizar búsquedas bibliográficas interdisciplinares y analizar la asiduidad con la que han sido citados los artículos en otras fuentes especializadas. Su uso se ha generalizado y ha ganado una gran popularidad entre los académicos (Xiao y Smith, 2008; Law et al., 2009). Por todo lo anterior se decidió utilizar Google Scholar y SCOPUS con el objetivo de medir el impacto de las aportaciones realizadas por los académicos. En el caso de Google Scholar, el número total de citas recibidas por cada artículo se obtuvo a partir de la función "Citado por".

Pese al diverso número de investigaciones realizadas sobre el análisis de la productividad investigadora de los académicos, en la actualidad no existente consenso en torno a su evaluación (Law y Chon, 2007; Severt et al., 2009). En cuanto a la medición de aspectos cuantitativos, las metodologías más relevantes e influyentes en relación a la investigación que llevaremos a cabo son el método de conteo por casos y el método fraccional.

El método de conteo por casos ("The instance counting method") ha sido utilizado tradicionalmente por un gran número de autores (Sheldon, 1991, Jogaratnam et al., 2005; Severt et al., 2009) y se basa en el conteo de la frecuencia en el que un determinado investigador o universidad participa activamente en la muestra de revistas objeto de estudio. Así, en un artículo escrito por 3 autores de diferentes universidades, siguiendo este método se deberá otorgar un punto a cada autor y un punto a cada universidad, lo que puede llevar como afirma Park et al. (2011) a que el peso de los autores individuales disminuya, y aumente la valoración de aquellos que han publicado un mayor número de artículos pero junto con más coautores. 
Por otro lado, el método fraccional ("Fractionals coring method") utilizado por Mathieu y McConomy (2003); Zhao y Ritchie (2007); Park et al. (2011) surge como una alternativa más objetiva que intenta eliminar las limitaciones del método anterior. En este caso hablamos de un método proporcional en el que a un artículo realizado por 3 autores pertenecientes a 3 universidades diferentes se le otorgaría 1/3 puntos a cada autor y $1 / 3$ puntos a cada universidad. La ventaja de este método radica principalmente en la proporcionalidad de la medición, siendo el número total de autores y universidades igual al de artículos publicados.

La literatura por tanto difiere en la selección del método que mida con mayor precisión las contribuciones de los académicos. Por ello, y siguiendo a Sheldon (1991), utilizaremos ambas metodologías en la medición para posteriormente comparar los resultados y medir la productividad y contribuciones de autores, países e instituciones académicas.

\section{RESULTADOS}

En primer lugar se muestran un conjunto de tablas descriptivas que tratarán de facilitar la comprensión del estado de la investigación científica sobre turismo en las instituciones académicas Argentinas y Chilenas.

Pese a que el eje central del estudio gira en torno al análisis de los datos obtenidos a través de la revisión de las 31 revistas científicas seleccionadas, es necesario incorporar otro tipo de indicadores que ayuden a obtener una visión global de la investigación científica a nivel mundial. El Scimago Journal\& Country Rank es un recurso online de acceso abierto para la evaluación y análisis de revistas científicas y países utilizando datos de publicación y citación de alrededor de 16.000 revistas indexadas por la base de datos Scopus de Elsevier. En este caso, se han seleccionado los artículos entre 2006 y 2010 dentro de la categoría SJR "tourism, leisure and hospitality management", para posteriormente obtener la gráficas que se presentan a continuación.

A continuación, en la gráfica 1 se muestra la evolución de las 8 principales regiones en relación a la productividad científica, destacando Norte América con un imparable progreso en el último lustro, seguida por la región del Pacífico y Europa Occidental. La productividad de las demás regiones es casi imperceptible por lo cual se generó la gráfica 2 para poder apreciar la evolución de las regiones con menor productividad. En particular, Latinoamérica ha mantenido un volumen de producción muy discreto con una media de 2 artículos anuales. Estos datos deben ser tomados con cautela, dado que la mayoría de las revistas seleccionadas por Scopus son de habla inglesa, por lo que la participación de revistas Latinoamericanas en el análisis es casi nula. 


\section{Gráficas 1 y 2. Evolución de la investigación en turismo por áreas geográficas (SJR).}
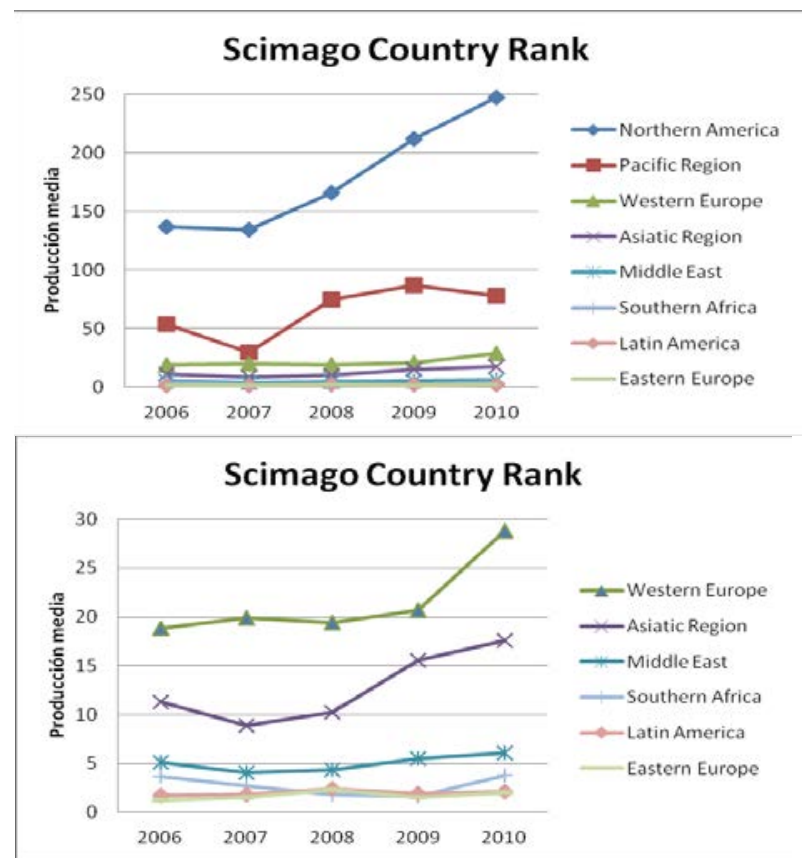

La gráfica 3 muestra la productividad científica en turismo de los países iberoamericanos con mayor volumen de producción entre 2006 y 2011. En relación al total de revistas analizadas, Brasil se encuentra a la cabeza, seguida muy de cerca por España. El hecho de que ambos países se encuentren en las primeras posiciones no es casual, ya que son las principales potencias iberoamericanas en investigación turística, aunque debe tenerse en cuenta que de las 31 publicaciones analizadas, 16 son Iberoamericanas y los resultados pueden verse distorsionados por el gran volumen de artículos que componen las ediciones de las 7 revistas Brasileñas y las 6 revistas españolas objeto de estudio. En la gráfica 4 se analiza de forma más detallada la evolución de Argentina y Chile en comparación con otras potencias iberoamericanas en investigación turística, reflejándose que ambos países han registrado una evolución intermitente con máximos en el año 2010 y un decrecimiento de la producción en el 2011. En cualquier caso, se observa un mayor volumen de producción por parte de los investigadores argentinos. 


\section{Gráfica 3 y 4. Evolución de la productividad científica en turismo en los países Iberoamericanos.}
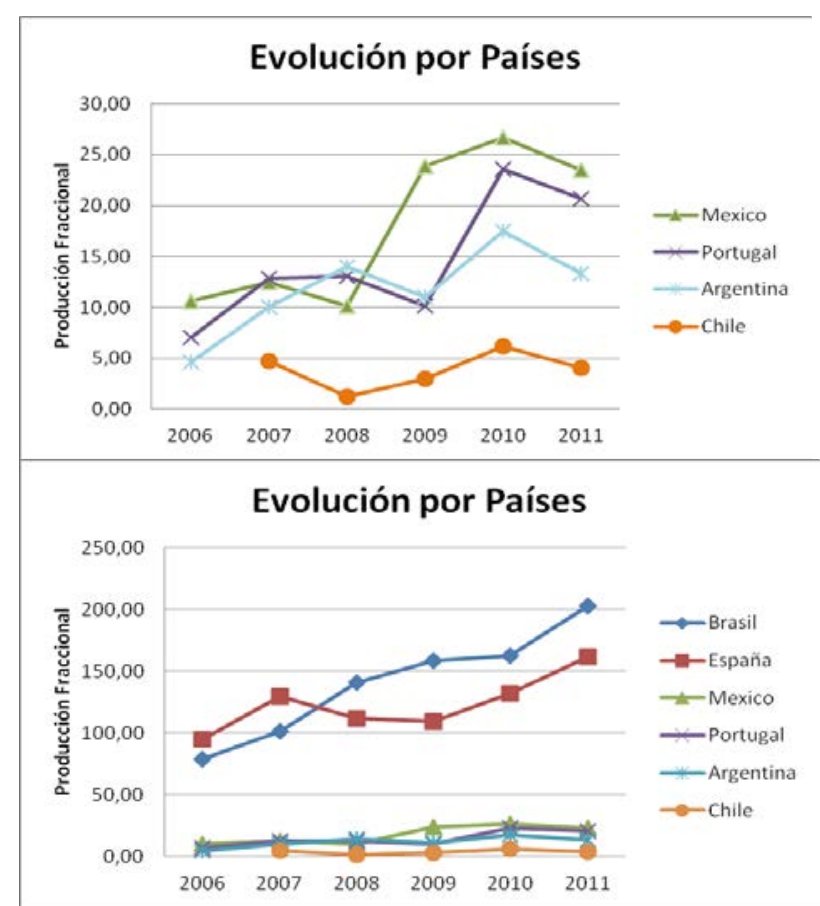

Una vez obtenida una visión global de la investigación turística en los países Iberoamericanos, es necesario analizar de forma individual su evolución.

A continuación se muestra en la tabla 2 y en la gráficas 5 y 6 la evolución y distribución de las instituciones Argentinas y Chilenas según el origen de las revistas (segmentándose en Brasileñas, Españolas, otras Latinoamericanas e Internacionales de habla inglesa). Puede observarse que la producción científica Argentina se ha centrado principalmente en "otras revistas latinoamericanas" y en las revistas brasileñas, siendo estas publicaciones las que engloban el 53,43\% y el 24,11\% de su producción total. Por otro lado las revistas españolas pese a la estabilidad de estos últimos años han presentando una fuerte disminución en el número de publicaciones en el año 2011 y la participación en revistas de habla inglesa ha sido prácticamente nula. Por último de los 2020 artículos objeto de estudio, sólo el 3,49\% han sido realizados por autores adscritos a instituciones Argentinas. La producción media anual ronda los 12 artículos y las instituciones argentinas representan el 3,5\% del total de la producción académica en turismo de Latinoamérica 
para las revistas analizadas.

En cuanto a la evolución de la producción Chilena, la gráfica 6 y la tabla 2 muestran una clara preferencia de los académicos chilenos por las publicaciones latinoamericanas, las cuales comprende el 90,88\% de su producción total. Pese al claro liderazgo de estas publicaciones su evolución ha sido intermitente, entre 0 y 6 artículos anuales. Por su parte, el número de investigaciones publicadas en revistas españolas e internacionales de habla inglesa representan el 5,20\% y el 3,90\%. La producción media anual ronda los 3 artículos y las instituciones chilenas representan casi el 1,0\% del total de la producción académica en turismo de Latinoamérica para las revistas analizadas.

\section{Gráficas 5 y 6. Evolución de la producción de las instituciones Argentinas y Chilenas por años según origen de las revista.}

Tabla 2. Presencia de las instituciones Argentinas y Chilenas por conjunto de revistas.

\begin{tabular}{|l|c|c|c|c|c|c|c|c|}
\hline & \multicolumn{2}{|c|}{$\begin{array}{c}\text { Producción } \\
\text { Total }\end{array}$} & \multicolumn{2}{c|}{ Artículos } & \multicolumn{2}{c|}{$\begin{array}{c}\text { \% Producción } \\
\text { por origen de } \\
\text { Revista }\end{array}$} & \multicolumn{2}{c|}{$\begin{array}{c}\text { \% Distribución de la } \\
\text { Producción }\end{array}$} \\
\hline $\begin{array}{c}\text { Origen de } \\
\text { Revista }\end{array}$ & ARG & CHL & ARG & CHL & ARG & CHL & ARG & CHL \\
\hline $\begin{array}{l}\text { Rev. } \\
\text { Latinoamericanas }\end{array}$ & 331 & 331 & 37,67 & 17,45 & $11,38 \%$ & $5,27 \%$ & $53,43 \%$ & $90,89 \%$ \\
\hline Rev. Brasileñas & 738 & 738 & 17,00 & 0,00 & $2,30 \%$ & $0,00 \%$ & $24,11 \%$ & $0,00 \%$ \\
\hline Rev. Españolas & 614,67 & 614,67 & 14,50 & 1,00 & $2,36 \%$ & $0,16 \%$ & $20,57 \%$ & $5,21 \%$ \\
\hline $\begin{array}{l}\text { Rev. } \\
\text { Internacionales }\end{array}$ & 335,98 & 335,98 & 1,33 & 0,75 & $0,40 \%$ & $0,22 \%$ & $1,89 \%$ & $3,91 \%$ \\
\hline \multicolumn{1}{|c|}{ Total } & $\mathbf{2 0 2 0}$ & $\mathbf{2 0 2 0}$ & $\mathbf{7 1}$ & $\mathbf{1 9}$ & $\mathbf{3 , 4 9 \%}$ & $\mathbf{0 , 9 5 \%}$ & $\mathbf{1 0 0 \%}$ & $\mathbf{1 0 0 \%}$ \\
\hline
\end{tabular}

Siguiendo con el análisis, la tabla 3 muestra la evolución de la producción de los académicos argentinos y chilenos en las diferentes revistas objeto de estudio. La revista argentina "Estudios y Perspectivas en Turismo" ha sido la principal plataforma de difusión tanto para los académicos argentinos como chilenos, concentrándose en ella, respectivamente, el $42,55 \%$ y el 58,33\% de su producción total. La revista española "Pasos", y la "Revista Brasileña de Pesquisa em Turismo" también han adquirido gran relevancia para los investigadores argentinos, representando el $13,47 \%$ y el $12,76 \%$ de su producción. Mientras que para las instituciones chilenas, destaca la revista "Gestión Turística”, editada en Chile, con un 31,25 \% de su producción total.

Las tablas que se exponen a continuación muestran el número de contribuciones realizadas por las principales instituciones Argentinas y Chilenas entre el año 2006 y 2011, calculadas a través del método fraccional. El objetivo principal es el análisis de las contribuciones científicas de cada institución y la identificación de las instituciones líderes en la investigación turística. La no aparición de una determinada institución académica en el ranking, o el situarse en los últimos puestos de la clasificación, no significa necesariamente que la institución sea inferior sino que puede que la investigación en turismo no se encuentre entre sus principales actividades.

En relación a las instituciones argentinas, las contribuciones realizadas por las 10 primeras representan el 74,04\% del total de la producción. La universidad argentina más productiva durante el periodo analizado ha sido la Universidad de Buenos Aires, la cual ha mantenido unos niveles de producción estables, destacando el año 2011 donde duplicó sus resultados. Le siguen la Universidad Nacional del Comahue y la Universidad Nacional de Quilmes, en segundo y tercer puesto. En el caso de Chile, las instituciones 
mostradas en la tabla 5 representan el 93,75\% del total de la producción. Pese al reducido número de artículos publicados en el año 2011, es la Universidad Austral de Chile la que lidera el ranking, seguida por la Universidad de Talca. 
Tabla 3. Evolución de la producción Argentina y Chilena por revistas y años.

\begin{tabular}{|c|c|c|c|c|c|c|c|c|c|c|c|c|c|c|c|c|c|c|c|c|}
\hline \multirow{2}{*}{ 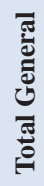 } & 思 & $\stackrel{2}{=}$ & $\stackrel{n}{0}$ & $\stackrel{F}{=}$ & $\stackrel{0}{0}$ & & & & & & & & $\stackrel{-1}{=}$ & $\stackrel{0}{\rightarrow}$ & & $\stackrel{\infty}{8}$ & & & $\begin{array}{l}\infty \\
0^{\infty}\end{array}$ & $\tilde{\Omega}^{2}$ \\
\hline & $\underset{\widetilde{r}}{\mathbb{Z}}$ & $\frac{5}{m}$ & $\stackrel{0}{\dot{m}^{-}}$ & $\begin{array}{l}0 \\
\tilde{e}^{n}\end{array}$ & $\tilde{\nabla}$ & $\stackrel{\circ}{\check{I}}$ & $\stackrel{0}{i}$ & $\stackrel{\circ}{\circ}$ & $\stackrel{0}{i}$ & $\stackrel{0}{\rightarrow}$ & $\stackrel{\circ}{\rightarrow}$ & $\stackrel{\circ}{i}$ & $\begin{array}{l}n \\
\pm \\
\pm\end{array}$ & in & $\begin{array}{l}\tilde{\omega} \\
\tilde{\sigma}\end{array}$ &  & $\stackrel{0}{\rightarrow}$ & $\stackrel{m}{0}$ & & $\begin{array}{l}n \\
2^{2}\end{array}$ \\
\hline \multirow{2}{*}{$\bar{\Xi}$} & 寻 & $m_{m}^{m}$ & & $\begin{array}{l}\infty \\
\sim \\
\sim\end{array}$ & $\stackrel{10}{0}$ & & & & & & & & & & & $\stackrel{\infty}{0}$ & & & $\begin{array}{l}\infty \\
0^{\prime}\end{array}$ & $\stackrel{\circ}{*}$ \\
\hline & U. & $\Leftrightarrow$ & $\stackrel{0}{\rightarrow}$ & $\begin{array}{l}0 \\
\infty\end{array}$ & & $\stackrel{\ominus}{+}$ & & $\stackrel{\circ}{n}$ & & & & $\stackrel{0}{\rightarrow}$ & $\tilde{m}$ & & $3_{0}^{m}$ & & & & & $\stackrel{2}{2}$ \\
\hline \multirow{2}{*}{$\stackrel{\ominus}{\overline{\mathrm{N}}}$} & 䍐 & ऊू & & $\stackrel{\sim}{\sim}$ & $\stackrel{0}{i}$ & & & & & & & & & & & & & & & तु \\
\hline & ب) & 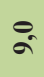 & & $\ddot{n}$ & & in & $\stackrel{0}{i}$ & $\stackrel{\circ}{i}$ & & $\stackrel{0}{\rightarrow}$ & & & लn & $\stackrel{0}{\rightarrow}$ & $\stackrel{12}{\sim}$ & & & & & $\stackrel{2}{5}$ \\
\hline \multirow{2}{*}{ 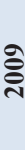 } & 寻 & $\stackrel{i}{i}$ & & $\stackrel{0}{-1}$ & $\stackrel{\circ}{-}$ & & & & & & & & $\stackrel{\theta}{=}$ & $\stackrel{0}{-}$ & & & & & & $\stackrel{\ominus}{m}$ \\
\hline & $\stackrel{y}{4}$ & $\hat{\theta}_{0}$ & & $\stackrel{0}{10}$ & $\stackrel{\circ}{\rightarrow}$ & $\stackrel{i}{i}$ & & $\stackrel{0}{-}$ & $\stackrel{\circ}{-}$ & & & & ले & $\stackrel{0}{-}$ & ì & & & & & $\stackrel{\ominus}{=}$ \\
\hline \multirow{2}{*}{$\stackrel{\infty}{\stackrel{\overbrace{}}{\circ}}$} & 具 & 3 & $\tilde{o}^{n}$ & $\stackrel{0}{-}$ & & & & & & & & & & & & & & & & 3 \\
\hline & 妾 & $\theta_{\infty}^{\infty}$ & $\stackrel{0}{i}$ & $\stackrel{0}{+}$ & $\stackrel{\circ}{i}$ & $\stackrel{\vec{m}}{m}$ & & $\stackrel{0}{i}$ & & & & $\stackrel{0}{\rightarrow}$ & $\ddot{m}^{\circ}$ & $\stackrel{0}{-}$ & $\stackrel{0}{i}$ & & & & & $\begin{array}{l}\stackrel{8}{ \pm} \\
\stackrel{ \pm}{ \pm}\end{array}$ \\
\hline \multirow{2}{*}{ હે } & 疍 & $\stackrel{\infty}{+}$ & & $\stackrel{m}{i}$ & $\stackrel{10}{\sim}$ & & & & & & & & & & & & & & & $\stackrel{\infty}{\infty}$ \\
\hline & U & $\stackrel{r}{f}$ & & $\stackrel{0}{m}$ & $\approx$ & $\stackrel{\theta}{m}$ & & $\stackrel{0}{-}$ & $\stackrel{0}{\rightarrow}$ & & $\stackrel{\circ}{\rightarrow}$ & & $\stackrel{i}{i}$ & $\stackrel{0}{\rightarrow}$ & $\stackrel{0}{-}$ & $\tilde{g}^{2}$ & & $\stackrel{m}{n}_{0}^{0}$ & & $\stackrel{\theta}{\hat{\theta}}$ \\
\hline \multirow{2}{*}{ ڤ్ํำ } & 寻 & & & & & & & & & & & & & & & & & & & $=$ \\
\hline & U. & $\stackrel{\ominus}{=}$ & & $\stackrel{0}{-1}$ & & & & & & & & & $\hat{i}$ & $\stackrel{0}{-}$ & $\hat{-}$ & $\stackrel{\theta}{=}$ & $\stackrel{0}{\rightarrow}$ & & & $F^{2}$ \\
\hline &  & 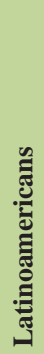 & 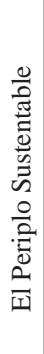 &  & 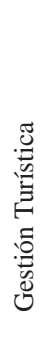 &  & 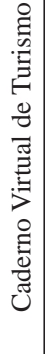 & 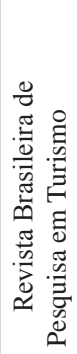 & 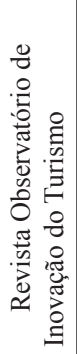 & 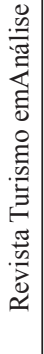 &  & 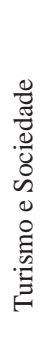 &  & 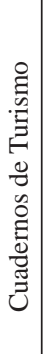 & 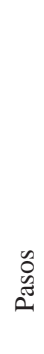 & 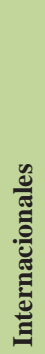 & 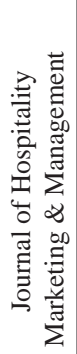 &  & 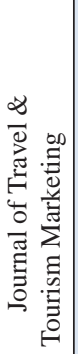 &  \\
\hline
\end{tabular}


Tabla 4. Evolución de la productividad de las instituciones Argentinas.

\begin{tabular}{|c|c|c|c|c|c|c|c|c|c|c|c|c|c|c|}
\hline \multicolumn{2}{|c|}{  } & İ & $m^{2}$ & 8 & $i^{2}$ & $F$ & $\stackrel{\rho}{+}$ & $\stackrel{P}{+}$ & $\stackrel{\rho}{\circ}$ & $\stackrel{\rho}{\circ}$ & in & สี & $\stackrel{\vec{\alpha}}{\underline{\alpha}}$ & $\stackrel{n}{\approx}$ \\
\hline \multirow{4}{*}{  } & 5 & : & $\stackrel{\circ}{+}$ & $\stackrel{\circ}{i}$ & $\stackrel{\circ}{+}$ & $\hat{m}$ & $\stackrel{9}{-}$ & & $\stackrel{n}{\rightarrow}$ & $\stackrel{\circ}{i}$ & $\stackrel{\circ}{=}$ & घิ & İ & $\stackrel{m}{m}$ \\
\hline &  & & $\stackrel{\circ}{-}$ & & & & & & & & & $\stackrel{8}{-}$ & 3 & 3 \\
\hline & 商 & $\stackrel{n}{\mathrm{~N}}$ & $\stackrel{i}{i}$ & $\stackrel{0}{-}$ & 照 & $\stackrel{\circ}{-}$ & $\stackrel{\circ}{\rightarrow}$ & & $\stackrel{2}{\rightarrow-1}$ & $\stackrel{\circ}{\rightarrow}$ & $\stackrel{\sim}{\rightarrow}$ & İ & in & $\stackrel{n}{ \pm}$ \\
\hline & 뜸 & $\dot{m}$ & : & $\stackrel{\circ}{\dot{m}}$ & $\stackrel{\circ}{\rightarrow}$ & & $\stackrel{i}{i}$ & $\stackrel{\circ}{+}$ & & & & $\stackrel{n}{\Rightarrow}$ & $m$ & $\stackrel{2}{\check{I}}$ \\
\hline \multirow{3}{*}{$\overline{\bar{z}}$} & 5 & $\vec{m}$ & ${ }_{i}$ & $\stackrel{\circ}{-}$ & & & & & $\stackrel{n}{2}$ & $\stackrel{\circ}{\rightarrow}$ & & 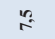 & $\because$ & $\therefore$ \\
\hline & 商 & & & & & & & & & & & 8 & 3 & 3 \\
\hline & $\stackrel{\propto}{\infty}$ & $\stackrel{\circ}{-}$ & & $\stackrel{\circ}{-}$ & & & & $\stackrel{i}{i}$ & & & & $f$ & 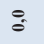 & $f$ \\
\hline \multirow{3}{*}{  } & 5 & $\stackrel{\circ}{-1}$ & & & $\stackrel{\dot{m}}{0}$ & & & & & & $\rightarrow$ & is & $\stackrel{8}{f}$ & $\therefore$ \\
\hline & 空 & $\stackrel{P}{\rightarrow}$ & & & 喿 & $\stackrel{\circ}{\rightarrow}$ & & & & & & 3 & $\stackrel{\odot}{-}$ & $m$ \\
\hline & 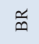 & & & $\stackrel{i}{i}$ & & & & $\stackrel{\circ}{\rightarrow}$ & & & & $\stackrel{P}{i}$ & $\stackrel{i}{i}$ & in \\
\hline \multirow{3}{*}{ ఏి } & 5 & $\stackrel{2}{\rightarrow}$ & & & & i & & & $\stackrel{\circ}{\rightarrow}$ & & & if & $\because$ & 8 \\
\hline & 商 & $\stackrel{\circ}{\rightarrow}$ & i & & & & & & & $\stackrel{\circ}{\rightarrow}$ & & $\stackrel{8}{\circ}$ & $\because$ & $\Leftrightarrow$ \\
\hline & 品 & & & & & & $\stackrel{\circ}{\rightarrow}$ & $\stackrel{\circ}{\rightarrow}$ & & & & i & $\stackrel{8}{8}$ & $\stackrel{i}{i}$ \\
\hline \multirow{3}{*}{ ఏે } & $\exists$ & & $\stackrel{\circ}{\rightarrow}$ & & $\stackrel{\circ}{\rightarrow}$ & $\stackrel{P}{\rightarrow}$ & $\stackrel{\circ}{=}$ & & & $\stackrel{\circ}{\rightarrow}$ & & is & $\stackrel{m}{m}$ & $\stackrel{\circ}{\infty}$ \\
\hline & 䔰 & & & $\stackrel{\circ}{\rightarrow}$ & & & $\stackrel{\circ}{=}$ & & & & $\stackrel{n}{8}$ & in & $\stackrel{n}{g}$ & $\stackrel{8}{\circ}$ \\
\hline & $\stackrel{\alpha}{x}$ & ${ }_{i}$ & & & $\stackrel{\circ}{\rightarrow}$ & & & & & & & $\stackrel{m}{\circ}$ & $\because$ & $\stackrel{\circ}{\circ}$ \\
\hline \multirow{4}{*}{ छे } & $\Xi$ & & $\stackrel{\circ}{i}$ & $\stackrel{\circ}{\rightarrow}$ & & $\hat{\circ}$ & & & & & & $\hat{i}$ & i & $f$ \\
\hline & $\underline{\geqq}$ & & & & & & & & & & & $\stackrel{8}{\circ}$ & 3 & $3_{0}^{2}$ \\
\hline & 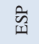 & & $\stackrel{\circ}{\rightarrow}$ & & & & & & & & $\stackrel{\circ}{\rightarrow}$ & i & $\stackrel{8}{8}$ & $\vec{i}$ \\
\hline & $\stackrel{\simeq}{m}$ & & 몽 & & & & $\stackrel{\circ}{\rightarrow}$ & & & & & $\because$ & $\because$ & $\stackrel{8}{i}$ \\
\hline \multirow{3}{*}{ క్ర } & 5 & $\stackrel{\circ}{\rightarrow}$ & & & & & & & & & & $\stackrel{1}{-}$ & $\therefore$ & $\stackrel{1}{-}$ \\
\hline & $\underline{\underline{z}}$ & & $\stackrel{0}{\rightarrow}$ & & & & & & & & & $\stackrel{2}{-}$ & 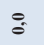 & $\stackrel{2}{-}$ \\
\hline & 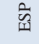 & no & & & & & & & $\stackrel{n}{\rightarrow}$ & & & i & $\hat{s}$ & $\hat{i}$ \\
\hline \multicolumn{2}{|c|}{ 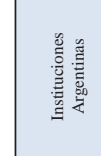 } & 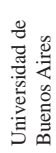 &  & 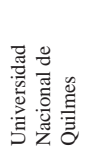 &  & 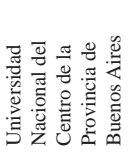 & 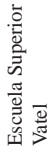 & 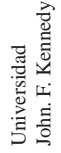 & 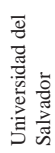 &  &  &  & 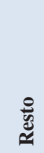 & 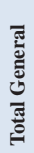 \\
\hline & & $\rightarrow$ & $N$ & $m$ & $\nabla$ & n & 0 & $n$ & $\infty$ & $\sigma$ & 우 & & & \\
\hline
\end{tabular}


Tabla 5. Evolución de la productividad de las instituciones Chilenas.

\begin{tabular}{|c|c|c|c|c|c|c|c|c|c|c|c|c|}
\hline & & 2007 & 2008 & \multicolumn{2}{|c|}{2009} & 2010 & \multicolumn{2}{|c|}{2011} & \multicolumn{3}{|c|}{ General } & \multirow{2}{*}{$\begin{array}{c}\text { Total } \\
\text { goneral }\end{array}$} \\
\hline & Chilenas & LT & LT & ESP & LT & LT & INT & LT & ESP & INT & LT & \\
\hline 1 & $\begin{array}{l}\text { Universidad } \\
\text { Austral de Chile }\end{array}$ & 1,8 & 0,3 & 1,0 & & 3,0 & & 0,5 & 1,0 & & 5,5 & 6,5 \\
\hline 2 & $\begin{array}{l}\text { Universidad de } \\
\text { Talca }\end{array}$ & 1,0 & & & & 1,0 & 0,8 & 0,8 & & 0,8 & 2,8 & 3,5 \\
\hline 3 & $\begin{array}{l}\text { Universidad de } \\
\text { Artes y Ciencias } \\
\text { Sociales }\end{array}$ & 1,0 & & & & & & & & & 1,0 & 1,0 \\
\hline 4 & $\begin{array}{l}\text { Universidad } \\
\text { Adolfo Ibáñez } \\
\text { Santiago }\end{array}$ & & 1,0 & & & & & & & & 1,0 & 1,0 \\
\hline 5 & $\begin{array}{l}\text { Universidad de } \\
\text { Concepción }\end{array}$ & & & & & 1,0 & & & & & 1,0 & 1,0 \\
\hline 6 & $\begin{array}{l}\text { Universidad de } \\
\text { Santiago de Chile }\end{array}$ & 1,0 & & & & & & & & & 1,0 & 1,0 \\
\hline 7 & $\begin{array}{l}\text { Programa } \\
\text { Patrimonio } \\
\text { Arqueológico Lo } \\
\text { Barnechea }\end{array}$ & & & & 1,0 & & & & & & 1,0 & 1,0 \\
\hline 8 & $\begin{array}{l}\text { Universidad } \\
\text { Austral Valdivia }\end{array}$ & & & & 1,0 & & & & & & 1,0 & 1,0 \\
\hline 9 & $\begin{array}{l}\text { Universidad } \\
\text { Católica de } \\
\text { Temuco }\end{array}$ & & & & & & & 1,0 & & & 1,0 & 1,0 \\
\hline 10 & $\begin{array}{l}\text { Pontificia } \\
\text { Universidad } \\
\text { Católica de Chile }\end{array}$ & & & & & 0,5 & & 0,5 & & & 1,0 & 1,0 \\
\hline & $\begin{array}{l}\text { Total 10 } \\
\text { Primeras }\end{array}$ & 4,8 & 1,3 & 1,0 & 2,0 & 5,5 & 0,8 & 2,8 & 1,0 & 0,8 & 16,3 & 18,0 \\
\hline & Resto & $\mathbf{0 , 0}$ & 0,0 & 0,0 & 0,0 & 0,7 & 0,0 & 0,5 & 0,0 & $\mathbf{0 , 0}$ & 1,2 & 1,2 \\
\hline & Total General & 4,8 & 1,3 & 1,0 & 2,0 & 6,2 & 0,8 & 3,3 & 1,0 & 0,8 & 17,5 & 19,2 \\
\hline
\end{tabular}


A continuación se detalla la distribución de la producción de las instituciones líderes por revistas. Como se observa en las tablas 6 y 7, la revista argentina Estudios y Perspectivas en Turismo se ha posicionado como la principal plataforma de difusión para la mayoría de las instituciones líderes, representando el 54,16\% de la producción de la Universidad de Buenos Aires, el $40 \%$ de la Universidad Nacional del Comahue y el $78,57 \%$ de la Universidad de Talca. Por otro lado, destaca entre las instituciones argentinas la revista española Pasos y la Revista Brasileira de Pesquisa em Turismo. En el caso de las instituciones Chilenas, existe una clara preferencia por la revista Estudios y Perspectivas en Turismo, representando el 58,33\% de su producción, seguida por la revista Gestión Turística editada por la Universidad Austral de Chile.

Tabla 6. Productividad de las instituciones Chilenas por revistas.

\begin{tabular}{|c|c|c|c|c|c|c|c|}
\hline & Revistas & $\begin{array}{c}\text { ESTUD. } \\
\text { PERSP. } \\
\text { TUR. }\end{array}$ & GESTUR & $\begin{array}{l}\text { CUAD. } \\
\text { TUR. }\end{array}$ & JTTM & PSUS & $\begin{array}{l}\text { Total } \\
\text { general }\end{array}$ \\
\hline 1 & Universidad Austral de Chile & 2,25 & 3,00 & 1,00 & & 0,25 & 6,50 \\
\hline 2 & Universidad de Talca & 2,75 & & & 0,75 & & 3,50 \\
\hline 3 & $\begin{array}{l}\text { Universidad de Artes y } \\
\text { Ciencias Sociales }\end{array}$ & 1,00 & & & & & 1,00 \\
\hline 4 & $\begin{array}{l}\text { Universidad Adolfo Ibáñez } \\
\text { Santiago }\end{array}$ & 1,00 & & & & & 1,00 \\
\hline 5 & Universidad de Concepción & 1,00 & & & & & 1,00 \\
\hline 6 & $\begin{array}{l}\text { Universidad de Santiago de } \\
\text { Chile }\end{array}$ & & 1,00 & & & & 1,00 \\
\hline 7 & $\begin{array}{l}\text { Programa Patrimonio } \\
\text { Arqueológico Lo Barnechea }\end{array}$ & & 1,00 & & & & 1,00 \\
\hline 8 & Universidad Austral Valdivia & 1,00 & & & & & 1,00 \\
\hline 9 & $\begin{array}{l}\text { Universidad Católica de } \\
\text { Temuco }\end{array}$ & 1,00 & & & & & 1,00 \\
\hline \multirow[t]{4}{*}{10} & $\begin{array}{l}\text { Pontificia Universidad } \\
\text { Católica de Chile }\end{array}$ & 0,50 & 0,50 & & & & 1,00 \\
\hline & Suma 10 Primeras & 10,50 & 5,50 & 1,00 & 0,75 & 0,25 & 18,00 \\
\hline & Resto & 0,70 & $\mathbf{0 , 5 0}$ & $\mathbf{0 , 0 0}$ & $\mathbf{0 , 0 0}$ & $\mathbf{0 , 0 0}$ & 1,20 \\
\hline & Total general & 11,20 & 6,00 & 1,00 & 0,75 & 0,25 & 19,20 \\
\hline
\end{tabular}


Tabla 7. Productividad de las instituciones Argentinas por revistas.

\begin{tabular}{|c|c|c|c|c|c|c|c|c|c|c|c|c|c|c|c|}
\hline & Revistas &  & 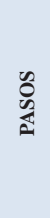 & 点 & 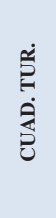 & 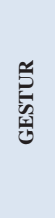 & $\overbrace{0}^{n}$ & 5 & 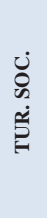 & 5 & $\sum_{E}$ & 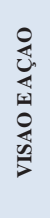 & 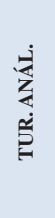 & 일 & 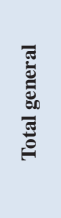 \\
\hline 1 & $\begin{array}{l}\text { Universidad de } \\
\text { Buenos Aires }\end{array}$ & 6,5 & 1,0 & 3,0 & 1,5 & & & & & & & & & & 12,0 \\
\hline 2 & $\begin{array}{l}\text { Universidad } \\
\text { Nacional del } \\
\text { Comahue }\end{array}$ & 3,0 & & 0,5 & 2,0 & 1,0 & & & & & 1,0 & & & & 7,5 \\
\hline 3 & $\begin{array}{l}\text { Universidad } \\
\text { Nacional de } \\
\text { Quilmes } \\
\end{array}$ & 2,0 & & 2,0 & 1,0 & & & & & & & & 1,0 & & 6,0 \\
\hline 4 & $\begin{array}{l}\text { Universidad } \\
\text { Nacional de } \\
\text { Mar del Plata }\end{array}$ & 4,0 & 0,5 & & & & & & 1,0 & & & & & & 5,5 \\
\hline 5 & $\begin{array}{l}\text { Universidad } \\
\text { Nacional del } \\
\text { Centro de la } \\
\text { Provincia de } \\
\text { Buenos Aires }\end{array}$ & 2,0 & 1,0 & & & 0,7 & 1,0 & & & & & & & & 4,7 \\
\hline 6 & $\begin{array}{l}\text { Escuela } \\
\text { Superior Vatel }\end{array}$ & 1,0 & 1,0 & 1,0 & & & & & & & & 1,0 & & & 4,0 \\
\hline 7 & $\begin{array}{l}\text { Universidad } \\
\text { John. F. } \\
\text { Kennedy }\end{array}$ & & & 2,0 & & & & & 1,0 & 1,0 & & & & & 4,0 \\
\hline 8 & $\begin{array}{l}\text { Universidad del } \\
\text { Salvador }\end{array}$ & & 1,0 & & 0,5 & 1,0 & 0,5 & & & & & & & & 3,0 \\
\hline 9 & $\begin{array}{l}\text { Universidad de } \\
\text { Palermo }\end{array}$ & 1,0 & 1,0 & & & & 1,0 & & & & & & & & 3,0 \\
\hline 10 & $\begin{array}{l}\text { Centro de } \\
\text { Investigaciones } \\
\text { y Estudios } \\
\text { Turísticos }\end{array}$ & 1,0 & 1,5 & & & & & & & & & & & & 2,5 \\
\hline & $\begin{array}{l}\text { Suma } 10 \\
\text { Primeras }\end{array}$ & 20,5 & 7,0 & 8,5 & 5,0 & 2,7 & 2,5 & $\mathbf{0 , 0}$ & 2,0 & 1,0 & 1,0 & 1,0 & 1,0 & $\mathbf{0 , 0}$ & 52,2 \\
\hline & Resto & 9,5 & 2,5 & 0,5 & $\mathbf{0 , 0}$ & 2,0 & 0,5 & 2,0 & $\mathbf{0 , 0}$ & 1,0 & $\mathbf{0 , 0}$ & $\mathbf{0 , 0}$ & $\mathbf{0 , 0}$ & 0,3 & 18,3 \\
\hline & Total general & 30,0 & 9,5 & 9,0 & 5,0 & 4,7 & 3,0 & 2,0 & 2,0 & 2,0 & 1,0 & 1,0 & 1,0 & 0,3 & 70,5 \\
\hline
\end{tabular}


La tabla número 8 presenta una relación de los investigadores adscritos a instituciones argentinas y chilenas que más participación han tenido en la producción de trabajos difundidos en las 31 revistas analizadas. En este análisis no se ha tenido en cuenta la relevancia de la revista a la hora de evaluar el liderazgo académico, y el ranking se ha elaborado en función del número de artículos en los que ha participado cada autor, a través del método fraccional.

En el caso de las instituciones argentinas, Maximiliano Korstanje fue el académico más productivo entre 2006 y 2011, y su claro liderazgo viene determinado por el número de publicaciones realizadas principalmente en revistas brasileñas y otras latinoamericanas. Le siguen Noemí Wallingre, Regina Schülter y Claudia Toselli con una participación fraccional de 3 artículos. En relación a los autores adscritos a universidades chilenas, Pablo Rebolledo Dujisin lidera el ranking con una participación fraccional de 1,5 artículos publicados exclusivamente en revistas latinoamericanas, seguido por Jorge Zamora con 1,3, Pablo Szmulewicz y otros autores. 
Tabla 8. Evolución de la productividad de los autores adscritos a instituciones argentinas por revistas.




Tabla 9. Evolución de la productividad de los autores adscritos a instituciones chilenas por revistas.

\begin{tabular}{|c|c|c|c|c|c|c|c|c|c|}
\hline \multirow{2}{*}{$\begin{array}{l}\text { Autores } \\
\text { Instituciones } \\
\text { Argentinas }\end{array}$} & \multirow{2}{*}{$\begin{array}{l}2007 \\
\text { LT }\end{array}$} & \multirow{2}{*}{$\begin{array}{l}2008 \\
\text { LT }\end{array}$} & \multicolumn{2}{|c|}{2009} & \multirow{2}{*}{$\begin{array}{l}2010 \\
\text { LT } \\
\end{array}$} & \multicolumn{2}{|c|}{2011} & \multirow[b]{2}{*}{$\begin{array}{l}\text { Total Suma } \\
\text { art. Fracción }\end{array}$} & \multirow[b]{2}{*}{$\begin{array}{l}\text { Total Suma } \\
\text { art. Absoluto }\end{array}$} \\
\hline & & & ESP & LT & & INT & LT & & \\
\hline $\begin{array}{l}\text { Pablo Rebolledo } \\
\text { Dujisin }\end{array}$ & & & & 1,0 & 0,5 & & & 1,5 & 2 \\
\hline Jorge Zamora & 0,5 & & & & 0,3 & 0,3 & 0,3 & 1,3 & 4 \\
\hline $\begin{array}{l}\text { Pablo Szmulewicz } \\
\text { E. }\end{array}$ & 1,0 & & & & & & & 1,0 & 2 \\
\hline $\begin{array}{l}\text { Daisy Núñez } \\
\text { Parrado }\end{array}$ & & & & & 1,0 & & & 1,0 & 2 \\
\hline $\begin{array}{l}\text { Ricardo Ibarra } \\
\text { Cofré }\end{array}$ & 1,0 & & & & & & & 1,0 & 1 \\
\hline Laura Nahuelhual & & & & 0,5 & 0,5 & & & 1,0 & 2 \\
\hline $\begin{array}{l}\text { ValmirMartins De } \\
\text { Oliveira }\end{array}$ & 1,0 & & & & & & & 1,0 & 1 \\
\hline $\begin{array}{l}\text { María Eugenia } \\
\text { Barril }\end{array}$ & 0,5 & & & & 0,3 & & & 0,8 & 2 \\
\hline $\begin{array}{l}\text { Rodrigo Fica } \\
\text { Pérez }\end{array}$ & & & & & 0,5 & & & 0,5 & 1 \\
\hline Rodrigo Hidalgo & & & & & & & 0,5 & 0,5 & 1 \\
\hline $\begin{array}{l}\text { Total } 10 \\
\text { primeros }\end{array}$ & 4,0 & $\mathbf{0 , 0}$ & 0,0 & 1,5 & 3,2 & 0,3 & 0,8 & $\mathbf{9 , 7}$ & 18,0 \\
\hline Total resto & 0,8 & 1,3 & 1,0 & 0,5 & $\mathbf{3 , 0}$ & 0,5 & 2,5 & 9,5 & 27,0 \\
\hline Total General & 4,8 & 1,3 & 1,0 & 2,0 & 6,2 & 0,8 & 3,3 & 19,20 & 45,0 \\
\hline
\end{tabular}

Con el objetivo de profundizar en el impacto de las investigaciones, se utilizó como herramienta principal de análisis la base de datos bibliográfica Scopus y Google Scholar. A continuación se presentan dos tablas por país con los autores y artículos líderes en relación al número de citas totales recibidas.

Como se puede observar en la tabla número 10, el autor que ostenta el primer puesto en el ranking argentino es Fiorella Guiliano, seguida por Maximiliano Korstanje con 28 y 17 citas respectivamente. A su vez, la tabla 11 referente a los autores adscritos a instituciones Chilenas, muestra que María Eugenia Barril y Jorge Zamora son los académico con mayor número de citas, ambas recibidas por las publicaciones realizadas 
en revistas latinoamericanas.

Si observamos las tablas 12 y 13, referente a los artículos de mayor impacto académico según el número de citas recibidas en Google Scholar, se aprecia que el artículo argentino con mayor difusión es "Algunas reflexiones sobre el turismo cultural" publicado en la revista española "Pasos", mientras que el artículo chileno más relevante fue "Turismo y vino. Un estudio formativo sobre la evolución de las rutas del vino en Chile” publicado en la revista argentina Estudios y Perspectivas en Turismo.

Tabla 10. Ranking de autores adscritos a instituciones argentinas por total de citas (Scopus y Google Scholar)

\begin{tabular}{|r|l|c|c|c|c|c|}
\hline & $\begin{array}{l}\text { Autores Instituciones } \\
\text { Argentinas }\end{array}$ & BR & ESP & INT & LT & Total general \\
\hline 1 & Fiorella Guiliano & & 17 & 11 & & 28 \\
\hline 2 & Maximiliano Korstanje & 6 & 0 & & 11 & 17 \\
\hline 3 & Lawrence Pratt & & 17 & & & 17 \\
\hline 4 & Claudia Toselli & & 14 & & 0 & 14 \\
\hline 5 & Claudia Alejandra Troncoso & & 0 & & 7 & 7 \\
\hline 6 & Rodolfo Bertoncello & & & & 7 & 7 \\
\hline 7 & Analía Almirón & & & & 7 & 7 \\
\hline 8 & Martín D. Mendieta & & 6 & & & 6 \\
\hline 9 & LailaVejsberg & & & & 6 & 6 \\
\hline 10 & Regina Schlüter & 0 & 5 & & 1 & 6 \\
\hline & Total 10 primeros & $\mathbf{6}$ & $\mathbf{5 9}$ & $\mathbf{1 1}$ & $\mathbf{3 9}$ & $\mathbf{1 1 5}$ \\
\hline
\end{tabular}


Tabla 11. Ranking de autores adscritos a instituciones chilenas por total de citas (Scopus y Google Scholar)

\begin{tabular}{|r|l|c|}
\hline & Autores Instituciones Chilenas & LT \\
\hline 1 & María Eugenia Barril & 4 \\
\hline 2 & Jorge Zamora & 4 \\
\hline 3 & Rodrigo Hidalgo & 3 \\
\hline 4 & Hugo Marcelo Zunino & 3 \\
\hline 5 & Ricardo Ibarra Cofré & 2 \\
\hline 6 & Pablo Rebolledo Dujisin & 2 \\
\hline 7 & Rodrigo Fica Pérez & 2 \\
\hline 8 & Edgardo Oyarzún Méndez & 2 \\
\hline 9 & Brenda Roman & 2 \\
\hline 10 & Laura Nahuelhual & 2 \\
\hline & Total 10 primeros & $\mathbf{2 6}$ \\
\hline
\end{tabular}

Tabla 12. Artículos de instituciones argentinas con mayor impacto en Google Scholar.

\begin{tabular}{|c|c|c|c|c|}
\hline $\mathrm{N}^{\circ}$ & Articulo & Autor & $\begin{array}{l}\text { Revista de } \\
\text { publicación }\end{array}$ & $\begin{array}{l}\text { Suma de } \\
\text { G o o g l e } \\
\text { Scholar }\end{array}$ \\
\hline 1 & $\begin{array}{l}\text { Algunas reflexiones sobre el turismo } \\
\text { cultural }\end{array}$ & Claudia Toselli & PASOS & 14,00 \\
\hline 2 & $\begin{array}{l}\text { Aportes de los viajes a las } \\
\text { ciencias sociales: Un relevamiento } \\
\text { bibliográfico para un análisis teórico }\end{array}$ & $\begin{array}{l}\text { M a x i m i l i a n o } \\
\text { Korstanje }\end{array}$ & GESTUR & 10,00 \\
\hline 3 & $\begin{array}{l}\text { Turismo, patrimonio y territorio. Una } \\
\text { discusión de sus relaciones a partir de } \\
\text { casos de Argentina }\end{array}$ & $\begin{array}{l}\text { Analía Almirón, } \\
\text { R o d o o l f o } \\
\text { B e r t o o c c e ll o, } \\
\text { Claudia Alejandra } \\
\text { Troncoso }\end{array}$ & $\begin{array}{l}\text { E S T U U D. } \\
\text { P E R S P. } \\
\text { TUR. }\end{array}$ & 7,00 \\
\hline 4 & $\begin{array}{l}\text { Reflexiones sobrelaconceptualización } \\
\text { de la competitividad de destinos } \\
\text { turísticos }\end{array}$ & $\begin{array}{l}\text { Rodrigo r. C. } \\
\text { González, Martín } \\
\text { D. Mendieta }\end{array}$ & $\begin{array}{l}\text { C U A D } \\
\text { TUR. }\end{array}$ & 6,00 \\
\hline 5 & $\begin{array}{l}\text { El proyecto turístico Barilochense } \\
\text { antes de Bustillo. Entre la prehistoria } \\
\text { del Parque Nacional Nahuel Huapi y } \\
\text { el desarrollo local }\end{array}$ & 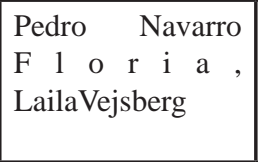 & $\begin{array}{l}\text { E S S T U D . } \\
\text { P E R S P. } \\
\text { TUR. }\end{array}$ & 5,00 \\
\hline & Total 5 primeros & & & 42,00 \\
\hline & Total general & & & 84,00 \\
\hline
\end{tabular}


Tabla 13. Artículos de instituciones chilenas con mayor impacto en Google Scholar.

\begin{tabular}{|l|l|l|l|l|}
\hline$N^{\circ}$ & Articulo & Autor & $\begin{array}{l}\text { Revista de } \\
\text { publicación }\end{array}$ & $\begin{array}{l}\text { Suma de } \\
\text { G o o gle } \\
\text { Scholar }\end{array}$ \\
\hline 1 & $\begin{array}{l}\text { Turismo y vino. Un estudio } \\
\text { formativo sobre la evolución de las } \\
\text { rutas del vino en Chile }\end{array}$ & $\begin{array}{l}\text { Jorge Zamora, } \\
\text { María Eugenia } \\
\text { Barril }\end{array}$ & $\begin{array}{l}\text { E S T U D . . } \\
\text { PERSP. TUR. }\end{array}$ & 4,00 \\
\hline 2 & $\begin{array}{l}\text { Negocios inmobiliarios en centros } \\
\text { turísticos de montaña y nuevos } \\
\text { modos de vida. }\end{array}$ & $\begin{array}{l}\text { R o d r i g o } \\
\text { Hidalgo, Hugo } \\
\text { Marcelo Zunino }\end{array}$ & $\begin{array}{l}\text { E S T U D . } \\
\text { PERSP. TUR. }\end{array}$ & 3,00 \\
\hline 3 & $\begin{array}{l}\text { La formación de guías de turismo } \\
\text { de montaña y alta montaña: El } \\
\text { caso Chileno }\end{array}$ & $\begin{array}{l}\text { P b l o } \\
\text { R e b l l e d o } \\
\text { D u j i s i n } \\
\text { Rodrigo Fica } \\
\text { Pérez }\end{array}$ & GESTUR & 2,00 \\
\hline 4 & $\begin{array}{l}\text { Áreas protegidas públicas y } \\
\text { privadas en el sur de Chile. } \\
\text { Caracterización del perfil de sus } \\
\text { visitantes d a }\end{array}$ & $\begin{array}{l}\text { Roman, Laura } \\
\text { Nahuelhual }\end{array}$ & PERSP. TUR. & 2,00 \\
\hline 5 & $\begin{array}{l}\text { Segregación socio-espacial en } \\
\text { ciudades turísticas. El caso de } \\
\text { Canela (RS), Brasil }\end{array}$ & $\begin{array}{l}\text { Ricardo Ibarra } \\
\text { Cofré }\end{array}$ & $\begin{array}{l}\text { E S T U D . } \\
\text { PERSP. TUR. }\end{array}$ & 2,00 \\
\hline Total 5 primeros & Total general & & 13,00 \\
\hline
\end{tabular}

En el siguiente apartado se analiza la distribución de las coautorías en la investigación turística argentina y chilena. Las siguientes tablas muestran la distribución de las coautorías en términos porcentuales, segmentando los datos por tipo de revistas (brasileñas, españolas, Internacionales de habla inglesa y otras de Latinoamérica) y país de la institución de origen del autor diferenciando entre Argentina o Internacional y Chile o Internacional. En la tabla 14 referente a los autores adscritos a instituciones argentinas, se aprecia que la gran mayoría de los artículos, cerca del 65\%, fueron publicados por un solo autor, el 26,8\% por dos, el 7\% por tres, y el 1,4\% por cinco autores. La publicación individual es por tanto la forma de participación más común, siendo utilizada por las instituciones argentinas en 46 de los artículos analizados. Esta forma contrasta con el estándar internacional, donde al coautoría tiene mayor peso proporcional, representando los trabajo de un único autor solamente el 31,4\%.Por el contrario, los autores adscritos a 
instituciones chilenas, prefieren la publicación conjunta, destacando las coautorías en el 47,6\% de los casos. La publicación individual supone el 41,3\%, y la de 3 o más autores el $38,1 \%$, destacando el peso de los trabajos con 4 autores, muy superior al estándar internacional. 
Tabla 14. Distribución de las coautorías de los autores adscritos a instituciones Argentinas

\begin{tabular}{|c|c|}
\hline 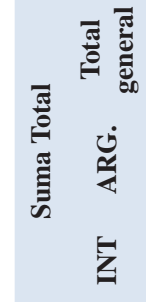 & 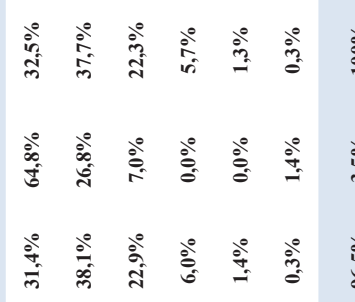 \\
\hline בَّ &  \\
\hline$\stackrel{\leftrightarrow}{\sharp}$ & 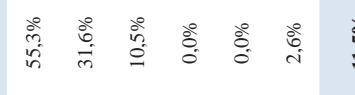 \\
\hline 气 & 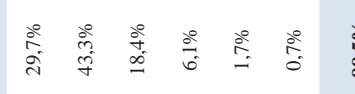 \\
\hline 番吉 & 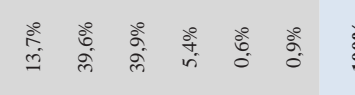 \\
\hline$\underline{\underline{z}}$ &  \\
\hline 气 & 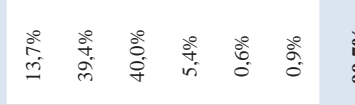 \\
\hline 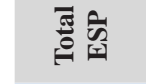 & 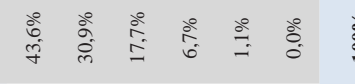 \\
\hline &  \\
\hline 气 & 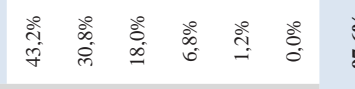 \\
\hline 吾 & 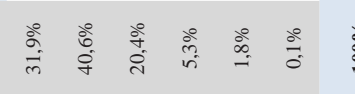 \\
\hline జ & 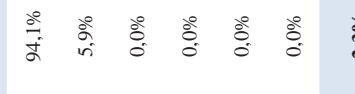 \\
\hline & 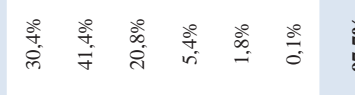 \\
\hline & \\
\hline
\end{tabular}


Tabla 15. Distribución de las coautorías de los autores adscritos a instituciones Chilenas

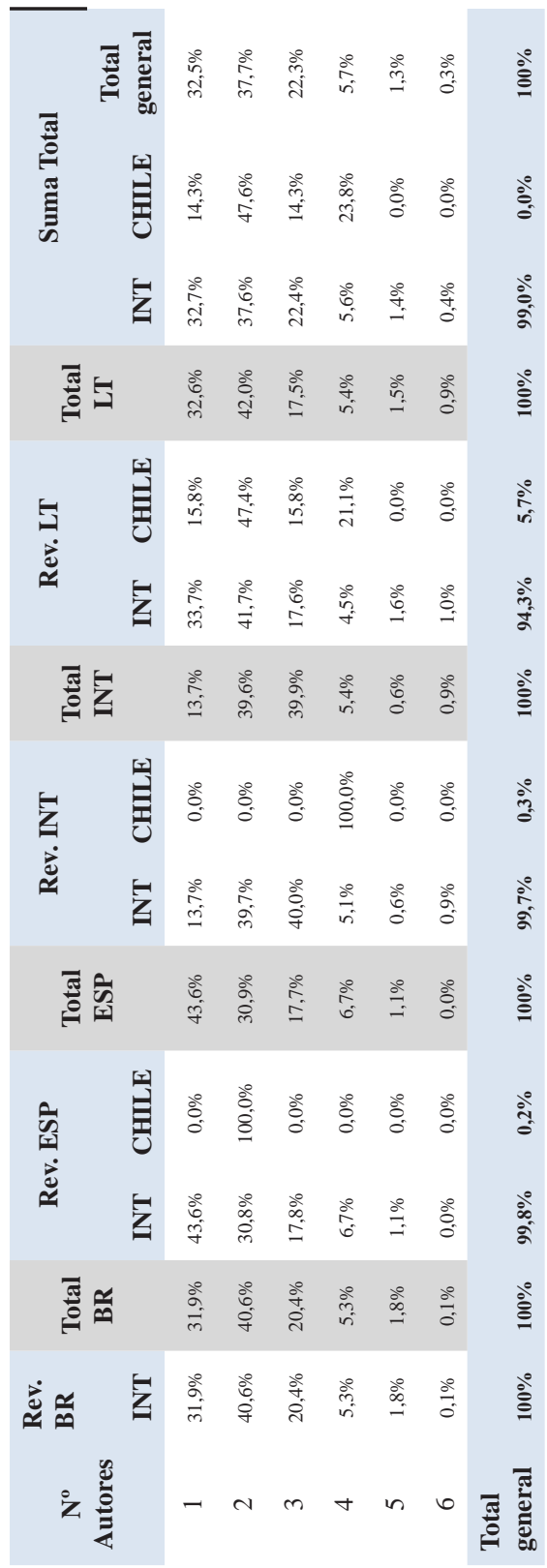


Por último, se ha procedido a cruzar los artículos difundidos por instituciones argentinas y chilenas con el ámbito geográfico de aplicación, es decir, analizando la región geográfica en la que se ha basado la investigación y que ha sido plasmada en el título del artículo o en sus palabras claves. En el 43,26\% de los artículos argentinos analizados no citó expresamente un área geográfica que constituyese la base del análisis, pero si analizáramos el 56,74\% restante como la producción total podríamos observar cómo la distribución geográfica se concentró claramente en Argentina en el 90\% de los artículos. En particular, las provincias de Buenos Aires y de Entre Ríos fueron las que despertaron una mayor atención entre los académicos. En el 41,67\% de los artículos chilenos se citó de forma explícita el ámbito geográfico de aplicación, apreciándose una clara preferencia por Chile y en especial por la Provincia de Aysén y la Metropolitana de Santiago.

Tabla 16. Ámbito geográfico de aplicación artículos argentinos

\begin{tabular}{lcc} 
Ámbito Geográfico & $\begin{array}{c}\text { Suma art. } \\
\text { Fracción }\end{array}$ & $\begin{array}{c}\text { \% art. } \\
\text { Fraccional }\end{array}$ \\
\hline Argentina & $\mathbf{3 6 , 0 0}$ & $\mathbf{9 0 , 0 0 \%}$ \\
Buenos Aires & 12,00 & $30,00 \%$ \\
Entre Ríos & 4,00 & $10,00 \%$ \\
Neuquén & 3,17 & $7,92 \%$ \\
Jujuy & 2,83 & $7,08 \%$ \\
Tierra de Fuego & 2,00 & $5,00 \%$ \\
Córdoba & 1,00 & $2,50 \%$ \\
Mendoza & 1,00 & $2,50 \%$ \\
$\quad$ Provincia del Chubut & 1,00 & $2,50 \%$ \\
$\quad$ Región del Norte & & \\
Grande Argentino & 1,00 & $2,50 \%$ \\
$\quad$ Región Patagónica & 0,50 & $1,25 \%$ \\
$\quad$ Sin Información & 7,50 & $18,75 \%$ \\
Italia & $\mathbf{2 , 0 0}$ & $\mathbf{5 , 0 0 \%}$ \\
Perú & $\mathbf{1 , 0 0}$ & $\mathbf{2 , 5 0 \%}$ \\
Brasil & $\mathbf{1 , 0 0}$ & $\mathbf{2 , 5 0 \%}$ \\
Total General & $\mathbf{4 0 , 0 0}$ & $\mathbf{1 0 0 , 0 0 \%}$
\end{tabular}


Tabla 17. Ámbito geográfico de aplicación de artículos chilenos

\begin{tabular}{lcc} 
Ámbito Geográfico & $\begin{array}{c}\text { Suma art. } \\
\text { Fracción }\end{array}$ & $\begin{array}{c}\text { \% art. } \\
\text { Fraccional }\end{array}$ \\
\hline Chile & $\mathbf{7 , 0 0}$ & $\mathbf{8 7 , 5 0 \%}$ \\
Aysén & 1,50 & $18,75 \%$ \\
Metropolitana de Santiago & 1,00 & $12,50 \%$ \\
Cautín & 0,50 & $6,25 \%$ \\
Región de los Ríos & 0,33 & $4,17 \%$ \\
Provincia del Ranco & 0,25 & $3,13 \%$ \\
Región del Maule & 0,25 & $3,13 \%$ \\
$\quad$ Sin Información & 3,17 & $39,58 \%$ \\
Brasil & $\mathbf{1 , 0 0}$ & $\mathbf{1 2 , 5 0 \%}$ \\
Total General & $\mathbf{8 , 0 0}$ & $\mathbf{1 0 0 , 0 0 \%}$ \\
\hline
\end{tabular}

\section{CONCLUSIONES}

El objetivo de este trabajo se centró en analizar la situación actual de la investigación en turismo en Argentina y Chile. Para ello, se realizó un estudio exhaustivo de todos los artículos publicados en 31 Revistas académicas de turismo (15 Internacionales de habla inglesa, 6 españolas, 7 brasileñas y 3 latinoamericanas), seleccionándose aquellas publicaciones realizadas por instituciones iberoamericanas, identificándose un total de 2020 artículos realizados por dichas instituciones en estas revistas. Así, del estudio de la evolución histórica realizada en el periodo 2006-2011 se observa un crecimiento discreto e intermitente de estos países de la investigación científica en este último lustro.

Las aportaciones argentinas han sido difundidas principalmente a través de "otras revistas latinoamericanas" y en revistas brasileñas, siendo estas publicaciones las que engloban el 53,43\% y el 24,11\% de su producción total. Por otra parte, del total de la producción Chilena en turismo, el 90,88\% de los artículos fueron publicados en "otras revistas latinoamericanas". En particular, la revista argentina "Estudios y Perspectivas en Turismo" fue la más popular entre los académicos de ambos países.

Por instituciones académicas, la universidad argentina más productiva durante el periodo analizado fue la Universidad de Buenos Aires, seguida por la Universidad Nacional del Comahue y la Universidad Nacional de Quilmes. En el caso chileno, la Universidad Austral de Chile lideró el ranking, seguida por la Universidad de Talca. 
Por otra parte, también se ha realizado un ranking de los autores con mayor aportación al aumento reciente de la investigación turística. Del análisis se concluye que los autores con mayor aportación al aumento de la investigación turística de Argentina son Maximiliano Korstanje, Noemí Wallingre, Regina Schülter y Claudia Toselli. En el caso de Chile, los autores más productivos fueron Pablo Rebolledo y Jorge Zamora. Por otra parte, la coautoría fue la forma de participación más común para los autores chilenos, siendo la producción individual la más popular entre los académicos argentinos.

Por último, en cuanto a la distribución geográfica, la producción argentina se centró en el análisis de las provincias argentinas, en particular, de las provincias de Buenos Aires y de Entre Ríos. En el caso de Chile, destacan especialmente por la Provincia de Aysén y la Metropolitana de Santiago.

Argentina y Chile han realizado un crecimiento significativo en la producción en investigación en turismo, habiendo generado numerosas revistas y artículos, que han situado a estos países entre los referentes de Sudamérica en la investigación turística. Esta investigación se ha centrado principalmente en publicaciones en revistas nacionales, dándose el salto recientemente a revistas internacionales (especialmente españolas y brasileñas) y quedando el reto de publicar en revistas de habla inglesa. Además, la difusión e impacto (citas) de las publicaciones realizadas es aún muy limitado. Se plantea así la necesidad de afrontar estrategias para continuar con la mejora de la producción científica en turismo.

En relación a las implicaciones académicas y prácticas, los análisis de este tipo de estudios sirven a las instituciones académicas para planificar futuras estrategias de fomento de la investigación, e incluso evaluar comparativamente la productividad de su personal, la posición de su institución a nivel global, o influir en la política de retribuciones, promociones y reparto de la docencia. Al mismo tiempo sirve como una guía para futuros estudiantes o personal investigador que desea conocer las instituciones con mayor prestigio para realizar estancias formativas o formalizar acuerdos (Jogaratnam, 2005; Mathieu \& McConomy, 2003; Zhao y Ritchie, 2007; Severt et al., 2009).

Este tipo de análisis además de actuar como un indicador de la productividad de las instituciones juegan un papel determinante en la carrera investigadora de muchos académicos (Ladkin y Weber, 2008; Jamal et al., 2008). En el pasado, los requisitos para ser promocionados dentro de las instituciones académicas eran menores, pero en la actualidad las universidades han aumentado el nivel de exigencia: publica o perece, requiriendo en algunos casos la producción de entre 12 y 16 publicaciones (Bowen, 2005), lo que ha motivado la búsqueda de revistas científicas con las menores barreras en la publicación y el mayor impacto. 
Por otra parte, el desarrollo de investigaciones relevantes por la comunidad académica podrían mejorar la imagen de las universidades o centros de investigación e incluso mejorar su financiación a través de la obtención de fondos estatales (Law y Chon 2007; Hall 2011).

Este trabajo, aunque ha seguido un riguroso análisis científico bibliométrico, no está exento de algunas limitaciones. Una de las principales limitaciones del estudio es la muestra de revistas seleccionadas. En general, los investigadores turísticos no se limitan a publicar sus estudios en revistas especializadas en turismo y hospitalidad (Law et al., 2009), como se ha comentado anteriormente el turismo es un área multidisciplinar, por lo que podrían haber investigaciones interesantes en turismo publicadas en otras revistas y por tanto no contabilizadas en este análisis.

Por otro lado, al analizar la productividad de las instituciones y autores, se tuvo en cuenta exclusivamente el número de artículos publicados en el periodo analizado, pero a la hora de realizar un listado de los investigadores líderes en el sector turístico, como sostiene Ryan (2005), se deberían tener en cuenta otros aspectos como las publicación de libros (algunos de gran contribución escritos por reconocidos investigadores), las supervisiones doctorales, investigaciones externas, captación de proyectos, capacidad de liderazgo etc.

Además, no se consideran las circunstancias del entorno de cada institución e investigador, que determinan de forma importante la productividad y posición en el ranking: número de investigadores por institución (que influye en su capacidad de producción, directa e indirectamente), la presencia de jóvenes investigadores, personal de apoyo, disponibilidad de fondos, estrategias académicas, atmósfera de investigación, disponer de una revista propia de la institución, etc. (Zhao y Ritchie, 2007; Lee y Law, 2011).

Por otra parte, existen limitaciones metodológicas, derivadas de la dificultad de plasmar adecuadamente la movilidad de los investigadores (incluso entre el momento de la publicación y el de realización del estudio) y la pertenecía de un mismo investigador a varias instituciones, y la posibilidad de incorporar errores por parte de los propios investigadores al analizar una base de datos de tanta complejidad. Además, el análisis de la literatura reafirma la idea de Zhao y Ritchie (2007) sobre la carencia de consenso en torno a la metodología de los rankings.

Finalmente, debería tenerse en cuenta que la cantidad de publicaciones no implica su calidad (Jogaratman et al., 2005). Jamal et al. (2008) destacan que los artículos deberían ser juzgados por su utilidad y no por el prestigio de la revista o el número de citas. La opinión de los expertos en el área permitirá obtener resultados válidos y 
fiables, ya que estarán más familiarizados con las publicaciones y su calidad (Pechlaner et al., 2004). Por otro lado Page (2005) invita a ser cautelosos en cuanto al volumen de producción científica ya que la producción masiva de publicaciones puede llevar a desarrollar investigaciones con unos niveles de rigurosidad y calidad inferiores a los esperados, y se debe tener en cuenta que el trabajo realizado por un autor se vería limitado si el artículo no tuviese ningún impacto en la comunidad académica (Law et al., 2009).

Pese a la limitaciones expuestas, es innegable que los ranking influyen en la capacidad de captación de financiación, captación de investigadores y estudiantes, promoción interna, etc. Por consiguiente, las instituciones deben considerar sus fortalezas y debilidades en relación a otros, y establecer estrategias de desarrollo de la investigación bien definidas. Anticipando la creciente competencia y globalización en el mundo académico, entender los ranking de productividad científica en turismo se convierte en un aspecto importante. Este estudio supone un primer esfuerzo en establecer un diagnostico general de la investigación turística en Argentina y Chile y desarrollar un ranking de instituciones e investigadores, que debe seguir desarrollándose en el futuro, dada la creciente importancia de esta nueva disciplina (turismo) en el mundo académico.

Como futuras líneas de investigación, se plantea analizar las relaciones de coautoría, los modelos de conformación de redes, y las prácticas y estrategias más exitosas al respecto. También analizar los temas y enfoques científicos de las diferentes investigaciones que se realizan, y aún más, la conexión de dichas temáticas con las necesidades actuales del turismo en Argentina y Chile. También se destaca la necesidad de analizar como potenciar la transferencia del conocimiento generado a la industria y la co-creación del mismo. Así mismo, se necesita mayor investigación sobre cómo gestionar y desarrollar con éxito la carrera investigadora y la excelencia en la investigación turística. Por último, parece adecuado realizar un análisis comparativo de la investigación en turismo en Argentina y Chile (contenido, metodología, etc.) en relación a la investigación turística internacional, y detectar posibles diferencias significativas al respecto.

Finalmente, destacar algunas estrategias de mejora de la producción científica en turismo que se derivan de este estudio. Al objeto de mejorar el desempeño de las universidades, es necesaria la publicación de sus estudios en revistas con factor de impacto (Mathieu \& McConomy, 2003), que son por tanto las que reciben más citas, lo que plantea el reto de alcanzar un mayor volumen de investigación en revistas internacionales de habla inglesa. Al objeto de abordar este último reto, parece necesario, entre otros, potenciar la formación académica e investigadora, los incentivos a las publicaciones de prestigio en la carrera profesional, la captación de talento internacional, las políticas de apoyo a la investigación (personal de apoyo, bases de datos de revistas, etc.), y el fomento de las redes internacionales con socios que aporten excelencia en investigación turística.

En cuanto al resultado del análisis del estudio relacionado con las colaboraciones 
en artículos (co-autorías), se puede intuir una falta de consolidación de equipos de investigación de turismo, siendo necesarios equipos consolidados para poder abordar investigaciones en revistas de prestigio de habla hispana e inglesa. Esto se puede fomentar con la creación de proyectos estructurantes y la creación de redes iberoamericanas de investigación en turismo. La colaboración entre académicos es muy beneficiosa, y así lo evidencia el hecho de que los investigadores con mayor número de colaboraciones tienden a ser los más productivos en el área (Racherla y Hu, 2010). Es importante destacar que el dominio de los países de habla inglesa en las publicaciones turísticas, no es sólo y únicamente debido al idioma, sino que también se sustenta en la calidad y tradición de los programas de doctorado en turismo en estos países, atrayendo talento internacional; la tradición histórica del turismo en estos países que con un entorno favorable posibilitó la aparición de jóvenes académicos en turismo; y la creación y desarrollo de prestigiosas revistas internacionales en inglés que atraen la difusión de todos los investigadores del mundo, y otorga ventaja a los nativos en este idioma (Zhao y Ritchie, 2007).

En relación a los resultados del ámbito geográfico de las investigaciones, estas se centran prácticamente en su totalidad en Argentina y Chile. Esto supone una ventaja, por la necesidad en la academia de un mayor enriquecimiento que puede representar para el conocimiento turístico las investigaciones realizadas en regiones con diferentes contextos culturales, como es el caso de Sudamérica, ya que el análisis y en especial la difusión de los estudios realizados en estas regiones han sido prácticamente inexistentes (Svensson, Svaeri y Einarsen, 2009). Al mismo tiempo, también es una desventaja, ya que la investigación llevada a cabo por Fastoso y Whitelock 2011, muestra que las principales dificultades en la publicación de artículos sobre estas áreas geográficas en revistas de reconocido prestigio son el sesgo negativo entre los editores y revisores en torno a las investigaciones realizadas en América Latina, el fracaso de los autores en conceptualizar teóricamente la investigación y las barreras idiomáticas. Por consiguiente, parece apropiado continuar con investigaciones sobre Argentina y Chile y sus peculiaridades (con enfoques novedosos, y no con simples aplicaciones y réplicas de trabajos previos, que garanticen su aplicación e interés universal), contando con equipos ampliados, y simultáneamente, ampliar el horizonte geográfico de las investigaciones.

En términos generales, se puede señalar que el balance del estado dinámico de la investigación turística en Argentina y Chile es positivo. La comunidad científica en turismo se va consolidando poco a poco al igual que sus investigaciones, pero se hace necesario potenciar el tratamiento científico de las investigaciones en turismo (más allá de estudios de naturaleza descriptiva, con una investigación aplicada fundamentada en teorías), fomentar los recursos dedicados a la investigación turística, así como el reconocimiento académico, el impulso a redes y equipos potentes en investigación. Se requiere pues de un mayor interés institucional por el turismo, con una mejor cooperación entre la aún reducida comunidad científica en turismo, y la conformación de una mayor 
tradición científica y de un conjunto determinado de valores, creencias y estructuras flexibles en investigación turística, ya que la realidad actual de la investigación en turismo aún se posiciona por concebir la actividad turística como un campo de estudio, más que una disciplina. Este planteamiento sugiere continuar con este tipo de análisis de ranking y evaluación dinámica de la investigación en turismo, así como el apoyo decidido a las múltiples sugerencias indicadas en este trabajo.

\section{BIBLIOGRAFÍA}

Albacete Sáez, C. A. y Fuentes Fuentes, M. M. (2010) "Difusión de la Investigación Española sobre Turismo en Revistas Internacionales" Revista de Análisis Turístico, 9: 14-29.

Bowen, J. T. (2005) “Managing a Research Career” International Journal of Contemporary Hospitality Management, 17(7): 633-637.

Castellucci, D. (2001) "Diagnóstico del estado de la investigación turística en las universidades argentinas” Aportes y Transferencias, 1 (5): 95-111.

Chung, J. Y. y Petrick, J. (2011) “Doctoral Students' Research Productivity: An Analysis of Publications in Tourism and Hospitality Journals” Journal of Hospitality, Leisure, Sports and Tourism Education, 10 (1): 63-71.

Frechtling, D. C. (2004) “Assessment of tourism/hospitality Journals' Role in Knowledge Transfer: An Exploratory Study” Journal of Travel Research, 43 (2): 100107.

Hall, M. (2011) "Publish and Perish? Bibliometric Analysis, Journal Ranking and the Assessment of Research Quality in Tourism" Tourism Management, 32 (1): 16-27.

Jamal, T., Smith, B. y Watson, E. (2008) "Ranking, Rating and Scoring of Tourism Journals: Interdisciplinary Challenges and Innovations" Tourism Management, 29 (1): 66-78.

Jogaratnam, G., Chon, K., Mccleary, K., Mena, M. y Yoo, J. (2005) “An Analysis of Institutional Contributors to Three Major Academic Tourism Journals: 1992-2001” Tourism Management, 26 (5): 641-648.

Ladkin, A. y Weber, K. (2008) "Tourism and Hospitality Academics: Career Profiles and Strategies” Journal of Teaching in Travel and Tourism, 8 (4): 373-393.

Law, R. y Chon, K. (2007) “Evaluating Research Performance in Tourism and Hospitality: 
The Perspective of University Program Heads” Tourism Management, 28 (5): 1203-1211.

Law, R., Leung, R. y Buhalis, D. (2010) “An Analysis of Academic Leadership in Hospitality and Tourism Journals” Journal of Hospitality and Tourism Research, 34 (4): 455-477.

Law, R., Ye, Q., Chen, W. y Leung, R. (2009) "An Analysis of the most Influential Articles Published in Tourism Journals from 2000 to 2007: A Google Scholar Approach” Journal of Travel and Tourism Marketing, 26 (7): 735746.

Lee, H. A. y Law, R. (2011) "Research Productivity and Institutional Characteristics of Hospitality and Tourism Programs" Journal of Travel and Tourism Marketing, 28 (4): 432-450.

Mathieu, R. y Mcconomy, B. J. (2003) “Productivity in “Top-Ten” Academic Accounting Journals by Researchers at Canadian Universities" Canadian Accounting Perspectives, 2 (1): 43-76.

Mckercher, B., Law, R. y Lam, T. (2006) "Rating Tourism and Hospitality Journals" Tourism Management, 27 (6): 1235-1252.

OMT. (2012) “UNWTO. Tourism Highlights" http://www.unwto.org/facts/menu.html

Page, S. J. (2005) “Academic Ranking Exercises: So they Achieve Anything Meaningful? - A Personal View.” Tourism Management, 26 (5): 663-666.

Park, K., Phillips, W.J., Canter, D. y Abbott, J. (2011) "Hospitality and Tourism Research Rankings by Author, University, and Country using Six Major Journals: The First Decade of the New Millennium" Journal of Hospitality and Tourism Research, 35 (3): 381-416.

Pechlaner, H., Zehrer, A., Matzler, K. y Abfalter, D. (2004) “A Ranking of International Tourism and Hospitality Journals" Journal of Travel Research, 42 (4): 328332.

Rivera, M. A. y Upchurch, R. (2008) "The Role of Research in the Hospitality Industry: A Content Analysis of the IJHM between 2000 and 2005" International Journal of Hospitality Management, 27 (4): 632-640.

Ryan, C. (2005) "The Ranking and Rating of Academics and Journals in Tourism Research” Tourism Management, 26 (5): 657-662.

Severt, D. E., Tesone, D.V., Bottorff, T.J. y Carpenter, M.L. (2009) “A World Ranking 
of the Top 100 Hospitality and Tourism Programs” Journal of Hospitality and Tourism Research, 33 (4): 451-470.

Sheldon, P. J. (1991) “An Authorship Analysis of Tourism Research” Annals of Tourism Research, 18 (3): 473-484.

Svensson, G; Svaeri, S. y Einarsen, K. (2009) “'Empirical Characteristics’ of Scholarly Journals in Hospitality and Tourism Research: An Assessment" International Journal of Hospitality Management, 28(3): 479-483.

Xiao, H. y Smith, S. L. J. (2008) "Knowledge Impact an Appraisal of Tourism Scholarship" Annals of Tourism Research, 35(1): 62-83.

Xiao, H. y Smith, S. L. J. (2006) "The Making of Tourism Research: Insights from a Social Sciences Journal” Annals of Tourism Research, 33(2): 490-507.

Zhao, W. y Ritchie, J. R. B. (2007) "An Investigation of Academic Leadership in Tourism Research: 1985-2004” Tourism Management, 28(2): 476-490.

Recibido: $15 / 08 / 2012$

Aceptado: 23/11/2012

Arbitrado anónimamente. 